\title{
Kinematic fit in CMS \& the use of top quarks for calibration
}

\author{
Jan Heyninck* ${ }^{* \dagger}$ \\ Vrije Universiteit Brussel (Belgium) \\ E-mail: heyninck@cern.ch
}

As a first topic, a kinematic fit implementation is described as it is used in CMS at the LHC collider. The importance of a kinematic fit for the top quark mass measurement is illustrated. Not only is the resolution on the final mass estimator reduced significantly, the probability of the kinematic fit can also be translated in a strong separation power between correct and wrong jet pairings.

The two other topics covered in this contribution, the measurement of the b-tagging efficiency and the measurement of the inclusive jet energy scale of light quark jets, are both examples of how the abundantly produced top quark pairs can be used for calibration tasks.

In the first study a highly b-enriched jet sample is constructed from the fully leptonic as well as the semileptonic $t \bar{t}$ decays, using a likelihood ratio method. The possible systematic uncertainties on the estimated b-purity were evaluated, leading to an optimized estimate of the expected precision on the measurement of the b-jet identification performance.

In the second study the absolute jet energy scale of the two reconstructed light quark jets from the semileptonic $t \bar{t}$ decay $(t \bar{t} \rightarrow W b \bar{W} \bar{b} \rightarrow l \bar{v} b q \bar{q} \bar{b})$ was determined via the precisely measured Wboson mass. Even for an integrated luminosity of $0.5 \mathrm{fb}^{-1}$ the systematic effects, mainly pile-up, on the optimal inclusive jet energy shift dominate the statistical uncertainty.

A precise knowledge of both the b-tag efficiency and the jet energy scale will be of major importance for precision measurements (e.g. top quark mass) and a whole spectrum of new physics studies (e.g. Higgs-boson search).

International Workshop on Top Quark Physics

January 12-15, 2006

Coimbra, Portugal

\footnotetext{
* Speaker.

$\dagger$ I want to thank all CMS-colleagues giving me input and feedback to this contribution.
} 


\section{Event Simulation}

The three discussed distributions are based on Monte Carlo simulated events. All were generated with PYTHIA 6.2 as event generator [1]. A full GEANT-4 detector simulation was used to simulate the detector response to the $t \bar{t}$ final state. Low-luminosity pile-up (with an instantaneous luminosity of $2 \cdot 10^{33} \mathrm{~cm}^{-2} \mathrm{~s}^{-1}$ ) from minimum bias events was added.

\section{Fitting of Event Topologies with External Constraints [2]}

\subsection{Definition and Output of a Kinematic Fit}

The resolution of the measured objects in the final state of proton collisions at the Large Hadron Collider can be improved by forcing well-defined kinematic hypotheses on the event. For a given event hypothesis the measured as well as unmeasured quantities are supposed to fulfill certain kinematic constraints. Due to the uncertainties in the measured quantities, these constraints are not exactly fulfilled. The well-defined concept of non-linear least square fitting together with the application of Langrange Multipliers can be utilized to ensure that the measured as well as unmeasured quantities exactly fulfill the external constraints deduced from the event hypothesis. This procedure is usually referred to as a kinematic fit. This results not only in a $\chi^{2}$-value that can be interpreted as a probability the imposed event hypothesis was true, but also in improved estimators of the underlying kinematics for a given event.

\subsection{Using Lagrange Multipliers for a Non-Linear Least Square Fit}

Assuming we have $n$ measured parameters $\vec{y}$ (e.g. particles 4-vector estimators), $p$ unmeasured parameters $\vec{a}$ (e.g. undetected neutrinos) and they are supposed to fulfill $m$ constraints $\vec{f}$ as defined in (2.1). These requirements will by definition be fulfilled for the true parameters $\bar{a}$ and $\bar{y}$.

$$
\begin{aligned}
f_{1}\left(\bar{a}_{1}, \bar{a}_{2}, \ldots, \bar{a}_{p}, \bar{y}_{1}, \bar{y}_{2}, \ldots, \bar{y}_{n}\right) & =0 \\
f_{2}\left(\bar{a}_{1}, \bar{a}_{2}, \ldots, \bar{a}_{p}, \bar{y}_{1}, \bar{y}_{2}, \ldots, \bar{y}_{n}\right) & =0 \\
\vdots & \\
f_{m}\left(\bar{a}_{1}, \bar{a}_{2}, \ldots, \bar{a}_{p}, \bar{y}_{1}, \bar{y}_{2}, \ldots, \bar{y}_{n}\right) & =0 .
\end{aligned}
$$

In general the measured values $\vec{y}$ will not solve the constraints. In that case a set of corrections $\Delta \vec{y}$ have to be calculated, requiring the sum $\vec{y}^{\prime}=\vec{y}+\Delta \vec{y}$ to fulfill the constraints. In the same time, the weighted sum

$$
S(\vec{y})=\Delta \vec{y}^{T} \mathscr{V}^{-1} \Delta \vec{y}
$$

should be minimal with $\mathscr{V}$ being the covariance matrix of the measured parameters. A general method to determine local extrema of non-linear functions of many variables is the use of Langrange Multipliers. The likelihood is then defined as follows:

$$
L(\vec{y}, \vec{a}, \vec{\lambda})=S(\vec{y})+2 \sum_{k=1}^{m} \lambda_{k} f_{k}(\vec{y}, \vec{a})
$$




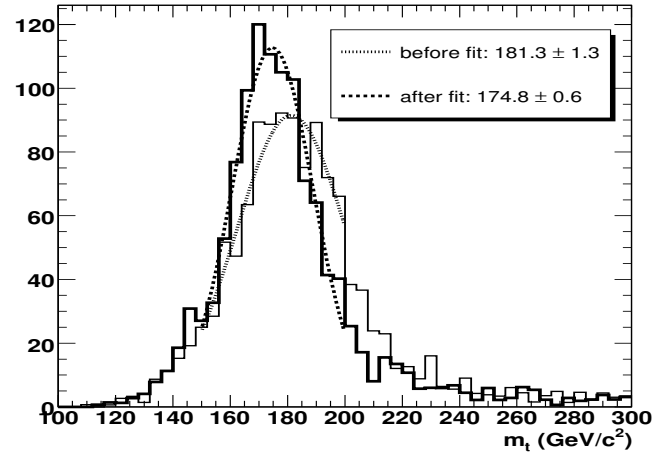

Figure 1: Distribution of the top quark mass spectrum with (red) and without (blue) the use of a kinematic fit. Only true jet combinations were used.

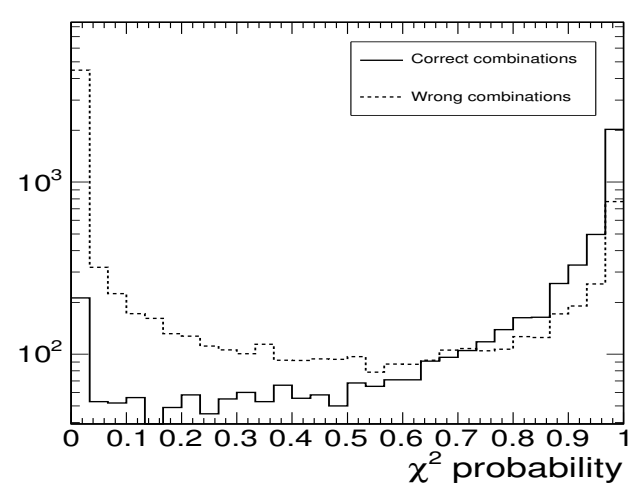

Figure 2: The probability of a knematic fit for correct and wrong jet combinations.

with $\vec{\lambda}$ the Lagrange Multipliers. The necessary condition for a local minimum of this function is then equivalent to the condition for a minimum of $S(\vec{y})$ under the constraints $f_{k}(\vec{y}, \vec{a})=0$. With linear constraints the solution can be found in one step, otherwise it has to be found iteratively by linearizing the constraint functions $f_{k}(\vec{y}, \vec{a})$ until all constraints are fulfilled within the required numerical precision.

\subsection{Importance of a Kinematic Fit for the Reconstructiona of Semileptonic $t \bar{t}$ events}

In the reconstruction of a semileptonically decaying $t \bar{t}$ pair, a kinematic fit can be applied to distinguish the correct from the wrong jet combinations, and to improve the resolution on the reconstructed top quark mass estimator. Two constraints are applied: one on the reconstructed hadronic $\mathrm{W}$ boson mass and one on the leptonic $\mathrm{W}$ boson mass. Both masses were forced to be equal to the current world average. The four-momenta of the jets and the hard muon, as well as an estimate of the transverse neutrino momentum component were passed as an input to the fit. The neutrino longitudinal momentum was not reconstructed but determined from the fit. The covariance matrices which represent the resolution of these variables are taken to be diagonal. The resolutions are differentiated as a function of the transverse momentum of the objects, hence, each individual object obtains a covariance matrix according to its transverse momentum. For the muon and the neutrino, a constant $E / \vec{p}$-ratio was required. After an identical event selection, the effect of this kinematic fit is illustrated in Figures 1 and 2. Figure 1 shows the significant reduction of the reconstructed top mass bias with respect to the generated $175 \mathrm{GeV} / \mathrm{c}^{2}$, as well as the improvement in resolution. In order to obtain the same statistical uncertainty, five times more statistics would be needed to equal the improvement of the kinematic fit. The probability of the kinematic fit for true and wrong jet combinations is plotted in Figure 2. This difference can e.g. be exploited in a combined likelihood ratio method. 


\section{Offline Calibration of $b$-jet Identification Efficiencies [3]}

\section{1 b-quark identification and b-tag calibration}

In general, b-jet identification techniques exploit the relatively large lifetime of B mesons, or the presence of leptons in the b-jet arising from $\mathrm{a} b \rightarrow \mathrm{cW}^{ \pm} \rightarrow \mathrm{c} \ell v$ decay. Some of the $\mathrm{b}$-jet identification algorithms have been studied in detail in CMS [4] on simulated events. On inclusive jet samples the CMS tracking system allows a typical b-tag efficiency of 50\%, for a rejection factor of $\sim 10$ for charm and $\sim 100$ for light-quark jets. The performance of these algorithms, however, is obtained from the models embedded in the simulations and hence will not exactly reproduce the reality in data. To calibrate the algorithmic efficiencies it is therefore crucial to measure the b-tagging efficiencies from the data.

\section{2 b-enriched jet samples from top quark events}

The enormous set of $t \bar{t}$-statistics expected at the LHC $\left(\sim 8\right.$ million for $\left.10 \mathrm{fb}^{-1}\right)$ can be used to select pure $b$-jet samples, neccessary to measure the b-tagging efficiency on data. Two tet decay channels are of particular interest and were investigated with simulated events:

- the semileptonic decay $\mathrm{t} \overline{\mathrm{t}} \rightarrow \mathrm{b}_{\bar{b}} \mathrm{qq} q^{\prime} \ell v$ : An isolated muon or electron, four jets and missing transverse momentum are required for the event selection. To fight the very large $\mathrm{W}^{ \pm}+$jets background $(116 \mathrm{nb})$ an extra requirement of at least one b-tagged jet is imposed;

- the fully leptonic decay $t \bar{t} \rightarrow b \bar{b} e v_{\mathrm{e}} \mu v_{\mu}$ : One muon and one electron of opposite charge, two jets and missing transverse momentum are required for an event to be selected. These selection criteria suppress very efficiently the large Z+jets (39 nb) background, and to a lesser extent the WW (190pb) and ZW (27 pb) backgrounds.

For each of these channels a trigger is provided by the isolated lepton.

In the case of the semileptonic events, the 12 possible jet associations were reconstructed with a kinematical fit imposing the $\mathrm{W}^{ \pm}$and top mass constraints. The best jet pairing was chosen by combining the fit results with several other observables into a combined likelihood ratio. In the case of fully leptonic events the kinematics are underconstrained due to the neutrinos, but still several event observables can be combined into a similar likelihood function.

In both cases, a cut can be made on the corresponding likelihood ratio to select well reconstructed events. The sample of jets associated with $\mathrm{t} \rightarrow \mathrm{b} \ell v$ decays in the semileptonic case, and the jet sample from both jets in the fully leptonic case, can be enriched in b-content with such a cut. In Figure 3 the b-jet purity for the different jet samples is shown as a function of the cut on the likelihood ratios. The semileptonic electron sample result is very similar to the one of the semileptonic muon sample.

\subsection{Systematic uncertainties on the sample purity}

In order to trust the sample purity obtained from simulations, the possible systematic uncertainties were evaluated [5]. It was shown that the relevant contributions to the systematic uncertainty come from initial and final state gluon radiation (ISR/FSR). In the fully leptonic case additionally the uncertainties on the signal over background cross sections ratio contribute (Figure 4). It 

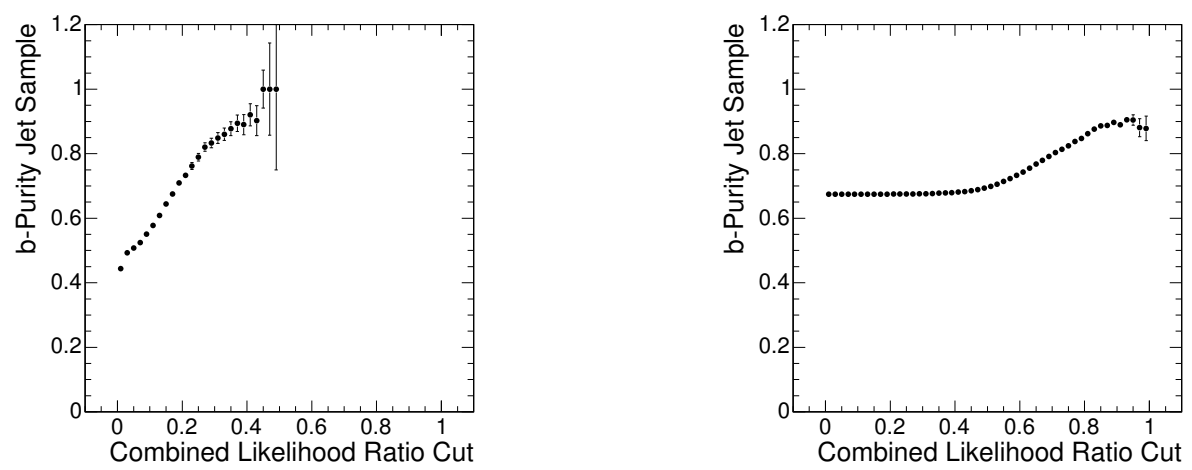

Figure 3: The b-jet purity of the different jet samples (semileptonic with muon (left) and fully leptonic (right)), as a function of the cut on the corresponding combined likelihood ratio.

is shown that the systematic uncertainties are reduced by cutting on the combined likelihood. Many other effects were investigated: the underlying event, pile-up, the luminosity, and the fragmentation model of light and b-quarks. All were shown not to contribute significantly. For semileptonic events, where mass constraints are applied on the events, the uncertainty on the jet energy scale, on one on the top mass, as well as the uncertainty due to the demanded b-tag of one jet in the event selection was found to be negligible.
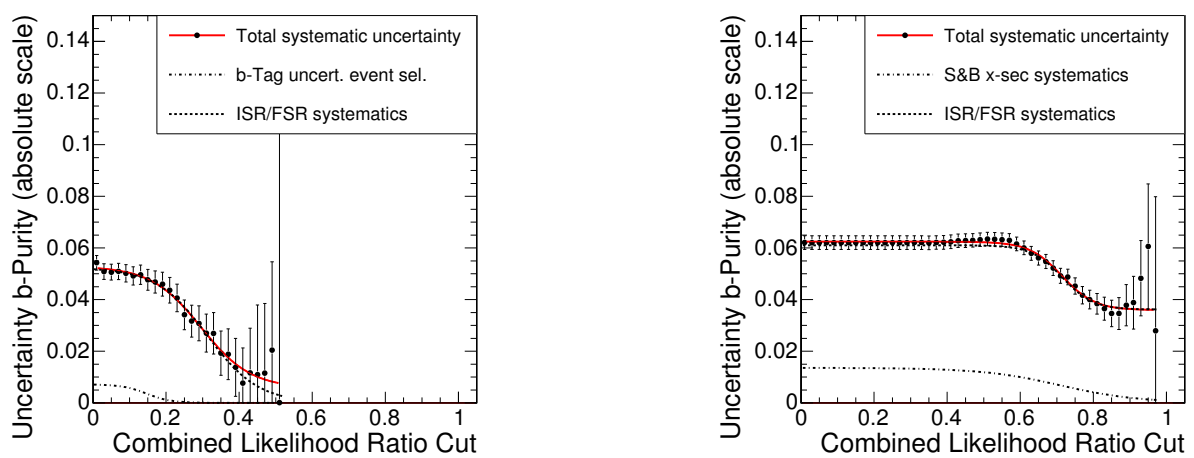

Figure 4: Systematic uncertainties on the b-purity of the different jet samples (semileptonic with muon (left) and fully leptonic (right)), as a function of the cut on the corresponding combined likelihood ratio.

\subsection{Measurement of $b$-tagging efficiencies on data}

On both jet samples the same technique is used to measure the b-tag efficiencies. Defining $x_{\mathrm{b}}$ as the b-purity of the jet sample, previously estimated from simulation, and $x_{\text {tag }}$ as the measured fraction of jets tagged by the b-tag algorithm, then the b-tagging efficiency

$$
\varepsilon_{\mathrm{b}}=\frac{1}{x_{\mathrm{b}}}\left[x_{\mathrm{tag}}-\varepsilon_{\mathrm{o}}\left(1-x_{\mathrm{b}}\right)\right],
$$

where $\varepsilon_{\mathrm{o}}$ is the mistag rate on the non-b jets in the sample. This mistag rate can be measured on data, but is for this study taken from simulation. 
It is possible to perform the measurement of $\varepsilon_{\mathrm{b}}$ for different cuts on the likelihood ratio. A point of minimal total uncertainty exists, and taken as the point where the actual estimation of $\varepsilon_{\mathrm{b}}$ is performed for the considered sample.

\subsection{Sample independent calibration}

The b-jet identification efficiencies depend on many sample specific jet parameter distributions, of which the most important ones are the transverse energy $E_{T}$ and the pseudorapidity $\eta$ of the jet. To make the calibration of the b-tag algorithms sample independent, the measurement of the efficiencies is performed as a function of these two parameters.

In Figure 5 the combined result from the three samples of this $E_{T}$ dependent measurement of $\varepsilon_{\mathrm{b}}$ is shown for jets in the barrel $(|\eta|<1.5)$ and endcap $(|\eta|>1.5)$ regions respectively, for $1 \mathrm{fb}^{-1}$ of data. b-tagging was performed with the default CMS algorithm. Any algorithm could have veen applied, however. Figure 6 shows the corresponding absolute and relative uncertainty expectations. The relative uncertainty on the b-tag efficiency measurement is expected to be about $6 \%$ in the barrel and between $7 \%$ and $12 \%$ in the endcaps for an integrated luminosity of $1 \mathrm{fb}^{-1}$.
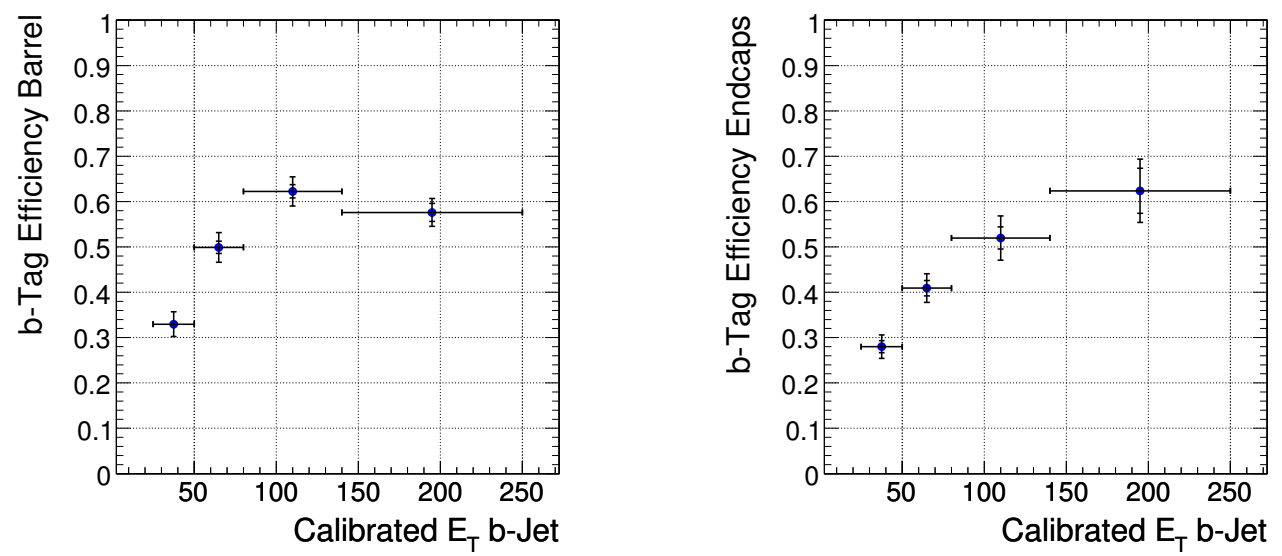

Figure 5: Theb-tag efficiency measurement, as a function of the jet $E_{T}$ for jets in the barrel $(|\eta|<1.5)$ (left) and endcaps $(|\eta|>1.5)$ (right).

\section{Light quark energy scale calibration using the $W$-mass constraint [6]}

\subsection{Introduction}

In the search for new physics or when aiming for precise measurements, the knowledge of the absolute energy scale of reconstructed jets originating from the quarks in the high- $\mathrm{Q}^{2}$ collision is crucial. One can invert the process and determine via the precisely measured $\mathrm{W}$ boson mass the absolute energy scale of the reconstructed jets in the $\mathrm{W} \rightarrow \mathrm{q} \overline{\mathrm{q}}$ decay. An estimate is presented of the precision that can be obtained using hadronic W bosons in the huge sample of $t \bar{t} \rightarrow b W \bar{b} W \rightarrow$ $b q \bar{q} \bar{b} l v$ events to be collected. 

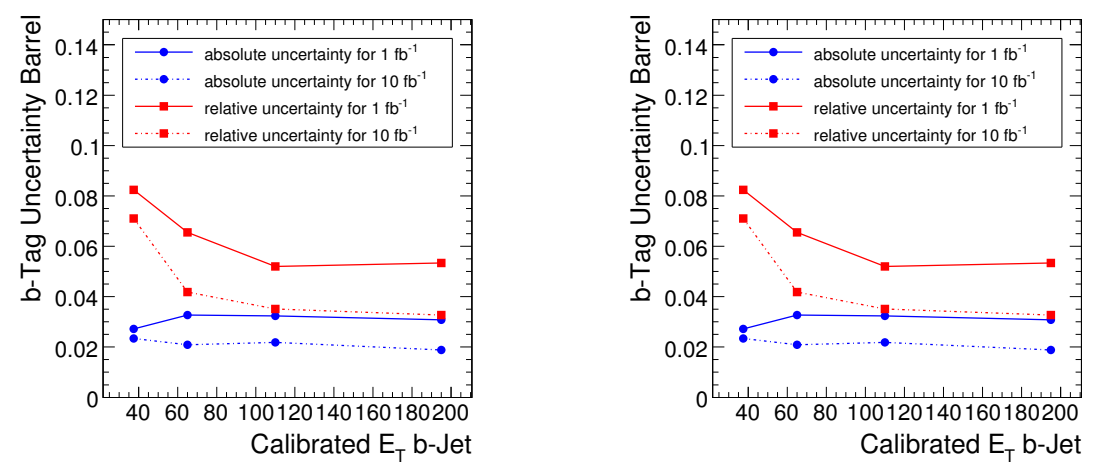

Figure 6: Uncertainty on the b-tag efficiency, as a function of the jet $E_{T}$ for jets in the barrel $(|\eta|<1.5)$ and endcaps $(|\eta|>1.5)$.

\subsection{Reconstruction of the events}

The jets in the final state are reconstructed with the Iterative Cone algorithm using an opening angle of $\Delta R=0.5 \mathrm{rad}$. Seeds for the cones where selected from all electromagnetic and hadronic calorimeter towers above a pseudo-rapidity dependent energy threshold determined from the average underlying event energy deposits [7].

The reconstructed jets were precalibrated with a standard CMS jet calibration. The applied $p_{T}$ and $|\eta|$-dependent scaling factors correct the non-unity average $E_{T}^{r e c} / E_{T}^{\text {gen }}$ ratio, when changing the jet definition from a reconstructed set of calorimeter towers to a set of generated particles from the vertex.
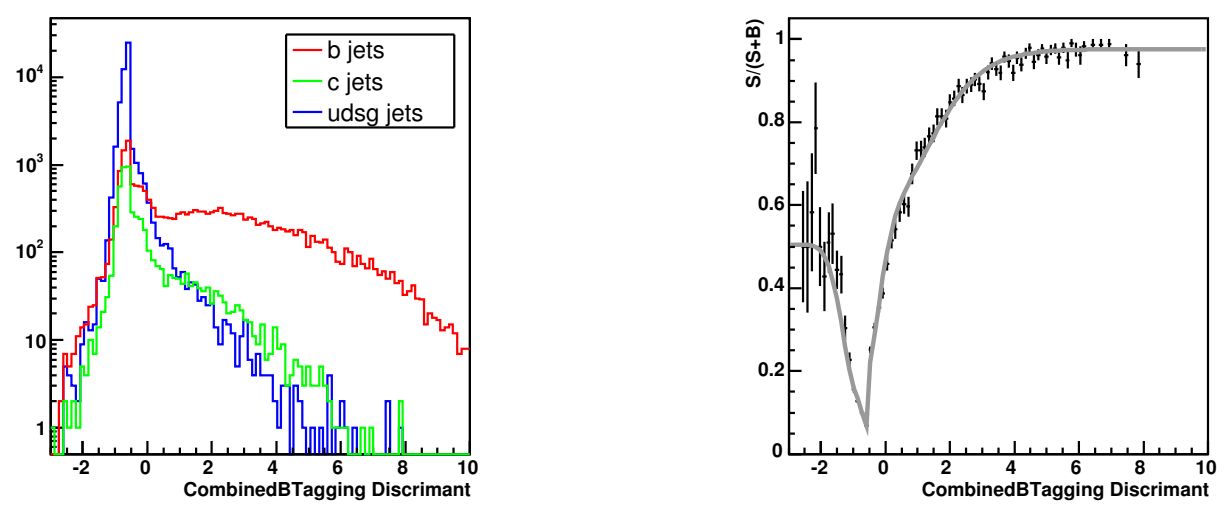

Figure 7: Distribution of the b-tag discriminant for jets originating from different flavoured quarks (left). On the right the b-tag probability (or likelihood ratio) as a function of the b-tag discriminant.

In order to discriminate between jets originating from the heavy b-quarks compared to the light quarks, a b-tag probability was constructed from the b-tag discriminant variable (Figure 7). With $P_{i}(x)$ the probability density functions of quark flavour $i$ in the dimension of the b-tag discriminant $x$, a likelihood ratio is constructed as

$$
L^{b}(x)=\frac{P_{b}(x)}{P_{b}(x)+P_{c}(x)+P_{\text {others }}(x)}
$$


which can be interpreted as the probability for the jet to originate from a b-flavoured parton.

The best hard muon candidate is selected amongst all reconstructed muons, using the likelihood ratio method described in [8]. This hard muon will pass the applied inclusive muon trigger[9] in $62 \%$ of the events.

Furthermore, the event is required to have exactly 4 calibrated jets with a $p_{T}$ above $30 \mathrm{GeV} / \mathrm{c}$ and reconstructed in the CMS tracker acceptance to ensure b-tagging $(|\eta|<2.4)$. Exactly two of these four jets need to have a b-tag probability $L^{b}$ larger than $60 \%$, the remaining two jets should not exceed a b-tag probability value of $30 \%$. It is also required that the cones of these four jets do not overlap in $(\eta, \phi)$ space. The reconstructed hard muon has to have a transverse momentum $p_{T}$ exceeding $20 \mathrm{GeV} / \mathrm{c}$ and pass the CMS tracker system.

Amongst the four selected jets in the final state, the two originating from the $\mathrm{W}$ boson decay are identified as the two jets having a b-tag probability below $30 \%$. This results in only two possible jet combinations to define the complete $t \bar{t}$ event. Events are selected if they have a jet combination which has a reconstructed hadronic top quark mass between 0 and $350 \mathrm{GeV} / \mathrm{c}^{2}$. This will reduce even further the process background.

When applying all mentioned event selection cuts, a signal-to-noise ratio of 3.8 is obtained. Most of the remaining background events are $t \bar{t}$ events with a $\tau$ lepton from $W \rightarrow \tau \nu_{\tau}$ in the final state. These events have however the same properties for the hadronic decaying $\mathrm{W}$-boson as the signal events, and should therefore not be considered as problematic background to the signal process. Due to the severe event selection cuts, few events remain from the analysed $\mathrm{W}+\mathrm{jets}$ process samples. Although the cross-section of the $\mathrm{W}+$ jets process is not negligible and this background should be considered with more adequate generators like AlpGen, it was omitted so far, and considered as a significant uncertainty in the estimate of the signal-to-noise ratio of about 0.5 .

\subsection{Estimator for the absolute Jet Energy Scale}

From the light quark jets identified as originating from the $\mathrm{W}$ boson decay, the $\mathrm{W}$ boson mass spectrum can be determined. In order to be more robust against outliers at large transverse momentum we only consider $\mathrm{W}$ decays for which both daughter jets have a transverse momentum $p_{T}$ below $120 \mathrm{GeV} / \mathrm{c}$. In the $\mathrm{W}$ boson mass window below $160 \mathrm{GeV} / \mathrm{c}^{2}$ the efficiency of obtaining the correct jets associated to the $\mathrm{W}$ boson decay reaches $80 \%$. The $\mathrm{W}$ boson mass spectrum is shown in Figure 8 for the event samples with the addition of pile-up collisions. The invariant $\mathrm{W}$ mass spectrum can be fitted with a simple Gaussian function $G\left(m_{W}, \sigma\right)$ and the mean value $m_{W}$ can be taken as an estimate of the reconstructed $\mathrm{W}$ boson mass. It is observed that the estimated $\mathrm{W}$ boson mass does not agree with the world average, $M_{W}=80.426 \pm 0.034 \mathrm{GeV} / \mathrm{c}^{2}$.

The $\mathrm{W}$ mass spectrum can also be constructed from jets which obtain an extra relative calibration shift $\Delta C$ (in $\%$ ) on their reconstructed energy scale $E_{j e t}$

$$
E_{\text {new }}=(1+\Delta C) \cdot E_{\text {jet }}
$$

resulting in a calibrated energy scale $E_{\text {new }}$. The direction of the jet is kept invariant for this procedure, while the magnitude of its momentum is rescaled in order to keep the E/p ratio of the jet invariant. Therefore one can fit several $m_{W}$ spectra with different values of $\Delta C$. The value of $\Delta C$ which results in a fitted $\mathrm{W}$ boson mass in agreement with the precise world average $M_{W}$ is the best 


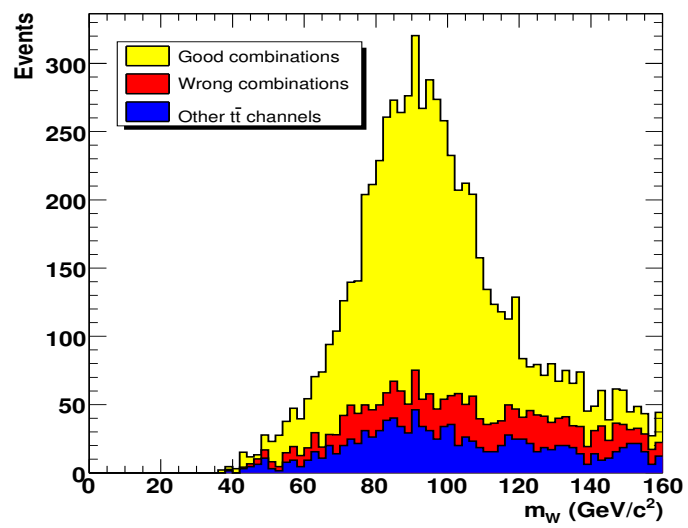

Figure 8: Distribution of the $\mathrm{W}$ boson mass spectrum. The combinatorial and process backgrounds are shown $\left(5.41 \mathrm{fb}^{-1}\right)$.

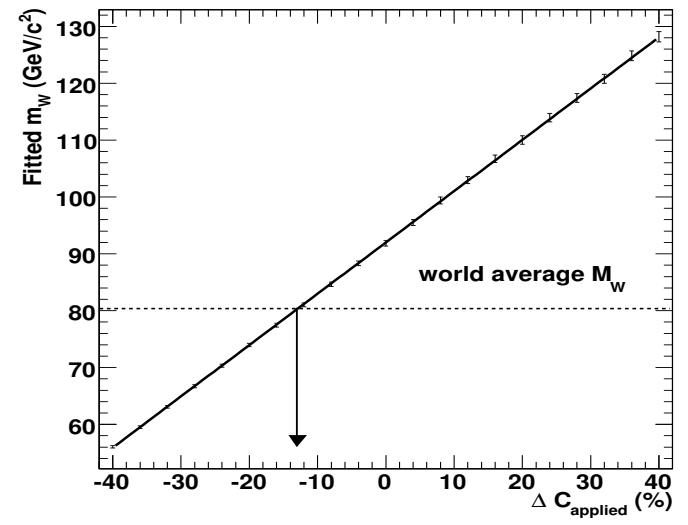

Figure 9: The dependency of the $\mathrm{W}$ boson mass estimator $m_{W}$ and the applied extra jet energy scale correction $\Delta C$ (for $\Delta C_{\text {true }}=-12.0 \%$ ).

estimate of the correction needed on the absolute jet energy scale for jets originating from light flavoured partons. For the nominal simulation samples described above, the relation between the fitted W mass $m_{W}$ and the applied jet energy scale correction $\Delta C$ is shown in Figure 9, resulting in an optimal value of $\Delta C=-12.7 \pm 0.3 \%$ (for $5.41 \mathrm{fb}^{-1}$ ). The uncertainty on $\Delta C$ is estimated from the uncertainty on the fitted $\mathrm{W}$ boson mass and the slope of the curve shown in Figure 9. This measured $\Delta C$ value can be compared with the true shift value, $\Delta C_{\text {true }}$, being defined as the gaussian fitted mean of $\left(E_{p}\right.$ arton/ $\left.E_{j} e t-1\right)$. Only non, overlapping, well-matched jets where used (spaceangle $\Omega$ (jet,parton) $<0.2 \mathrm{rad}$ ). The true shift value is found to be $\Delta C_{\text {true }}=-12.0 \%$, resulting in a bias $\Delta C_{\text {bias }}=\Delta C-\Delta C_{\text {true }}=-0.7 \pm 0.4 \%$.

The robustness of the bias $\Delta C_{\text {bias }}$ versus the criterion used for the jet-to-parton matching was checked and showed to be negligible, but $\Delta C_{\text {bias }}$ is found to be sensitive to the reconstructed opening angle of the W-decay $\Omega_{W}$. Selecting only events with a $\Omega_{W}$ higher than $1 \mathrm{rad}$ resulted in $\Delta C_{\text {bias }}=$ $-0.9 \pm 0.4 \%$. Furthermore, $\Delta C_{\text {bias }}$ will be enhanced by the fact that the jet energy scale correction $\Delta C$ depends on the kinematics of the $W \rightarrow q \bar{q}^{\prime}$ decays used. Therefore extentions of this inclusive method should be studied, for example by estimating the measured jet energy scale correction as a function of the $p_{T}$ of the jets.

\subsection{Possible systematic uncertainties}

The influence of pile-up is found to be $\Delta C$ (PU-noPU) $=-3.1 \%$. This was estimated by using simulated signal event samples with and without low luminosity pile-up collisions. This is the average effect on the energy scale of each reconstructed jet from the inclusion of pile-up collisions in-time with the bunch crossing containing the hard- $\mathrm{Q}^{2}$ event. With the advent of efficient pile-up subtraction methods, this difference should decrease.

The influence of the combinatorial background is estimated from a $\mathrm{W}$ boson mass spectrum with and without including this combinatorial background. The difference is found to be $\Delta C$ (BcknoBck $)=0.13 \%$. In a similar way the influence of including the other- $t \bar{t}$ process background is 
determined and a difference of $\Delta C($ Bck-noBck $)=0.17 \%$ is observed. The total systematic uncertainty on $\Delta C$ is conservatively estimated to be equal to $3.1 \%$ and completely dominated by the effect of pile-up.

\section{Conclusion}

- Kinematic Fit in CMS: It was demonstrated that the use of a kinematic fit in the reconstruction of the top quark mass in the semileptonic $t \bar{t}$ decay leads to a gain of a factor five in statistics. A significant reduction of the bias with respect to the generated top mass value was observed, and the importance of the probablity of the kinematic fit in choosing the correct jet combination illustrated.

- b-Jet Identification Efficiency Measurement using $t \bar{t}$-events: For $1 \mathrm{fb}^{-1}$ of integrated luminosity the combined relative accuracy on the b-identification efficiency of the considered algorithm is expected to be about $6 \%$ in the barrel region and between $7 \%$ and $12 \%$ in the endcaps of the detector.

- Light Quark Jet Energy Scale Calibration: With an efficient and well understood b-tagging algorithm, for $1 \mathrm{fb}^{-1}$ of integrated luminosity the statistical precision is already better than $1 \%$. The effect of the $\mathrm{W}+$ jets background process and the use of pile-up subtraction methods should be studied carefully.

\section{References}

[1] T.Sjöstrand, P.Edén, C.Friberg, L.Lönnblad, G.Miu, S.Mrenna and E.Norrbin, 'High-Energy-Physics Event Generation with PYTHIA 6.1', Computer Phys. Commun. 135 (2001) 238 (LU TP 00-30, hep-ph/0010017).

[2] J.D'Hondt e.a., 'Fitting of Event Topologies with External Kinematic Constraints in CMS', CMS Note 2006-023.

[3] S. Lowette, J. D'Hondt, J. Heyninck, P. Vanlaer, 'Offline Calibration of b-Jet Identification Efficiencies', CMS Note 2006-013.

[4] CMS Collaboration, Technical Design Report: The TriDAS Project, Volume II: Data Acquisition \& High Level Trigger, CERN/LHCC/2002-26, 2002.

[5] P. Bartalini, R. Chierici and A. De Roeck Guidelines for the Estimation of Theoretical Uncertainties at the LHC, CMS Note 2005/013.

[6] J. D'Hondt, S. Lowette, J. Heyninck, S. Kasselmann, 'Light quark jet energy scale calibration using

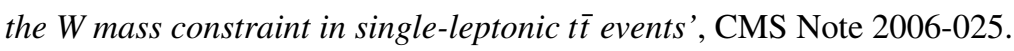

[7] A.Heister et al., 'Jet Reconstruction and Performance in the CMS Detector', CMS Analysis Note 2005/005.

[8] J.D'Hondt, J.Heyninck, S.Lowette, 'Electron and muon reconstruction in single leptonic ttbar events', CMS Note 2006-024.

[9] CMS Collaboration, 'Data Acquisition and High-Level Trigger', Technical Design Report, volume II. 